\title{
CORRESPONDENCE
}

\section{The 11th pitfall: thiamine deficiency}

CrossMark

\author{
Zaccaria Ricci ${ }^{i^{*}}$ (1) and Stefano Romagnoli
}

C 2018 Springer-Verlag GmbH Germany, part of Springer Nature and ESICM

Dear Editor,

We read with great interest the article by Hernandez et al. [1]. We think that, regarding lactate and glucose metabolism, the occurrence of thiamine deficit is worth mentioning since it is frequently overlooked in critically ill (septic) patients [2].

Thiamine (or vitamin B1) active form is thiamine pyrophosphate or thiamine diphosphate and represents a co-factor of pyruvate dehydrogenase, alpha-ketoglutaric acid dehydrogenase, and transketolase, three critical enzymes of carbohydrate metabolism. The first of these, pyruvate dehydrogenase, catalyzes the conversion of pyruvate to acetyl-CoA. Thus, glucose metabolism requires thiamine as a mandatory element to avoid lactate accumulation. In thiamine deficiency or depletion, lactic acid will accumulate to produce severe lactic acidosis, and thiamine supplementation implies a rapid lactate decrease (not clearance) [3].

Thiamine deficit may occur in critically ill patients in case of increased glucose metabolism (i.e., in septic states or post-surgical phases), sudden or aggressive nutrition delivery to malnourished patients (refeeding syndrome), or excessive removal (as during continuous renal replacement therapy, especially in case of high-dose prescription) [2]. Pediatric critically ill patients, especially in case of infection, have shown a thiamine deficit incidence of about $25 \%$ upon intensive care unit admission [2]. In any case, to avoid lactic acidosis secondary to thiamine deficiency, adequate supplementation with parenteral or enteral nutrition is crucial. In general, the daily maintenance requirement in adults ranges from 1.1 to $1.2 \mathrm{mg}$ orally and $3 \mathrm{mg}$ parenterally, but this dose can be increased up to $100-300 \mathrm{mg} /$ day in patients with signs of thiamine deficit [2].

\section{Author details \\ ${ }^{1}$ Department of Cardiology and Cardiac Surgery, Pediatric Cardiac Inten- sive Care Unit, Bambino Gesù Children's Hospital, IRCCS, Piazza S.Onofrio 4, 00165 Rome, Italy. ${ }^{2}$ Department of Anesthesia and Intensive Care, University of Florence, Azienda Ospedaliero-Universitaria Careggi, Florence, Italy.}

\section{Compliance with ethical standards}

Conflicts of interest

The authors declare they have no conflict of interest.

Received: 20 May 2018 Accepted: 5 June 2018

Published online: 22 June 2018

\section{References}

1. Hernandez G, Bellomo R, Bakker J (2018) The ten pitfalls of lactate clearance in sepsis. Intensive Care Med. https://doi.org/10.1007/s0013 4-018-5213-x

2. Manzanares W, Hardy G (2011) Thiamine supplementation in the critically ill. Curr Opin Clin Nutr Metab Care 14:610-617

3. Giacalone M, Martinelli R, Abramo A et al (2015) Rapid reversal of severe lactic acidosis after thiamine administration in critically ill adults: a report of 3 cases. Nutr Clin Pract 30:104-110 